\title{
Impact of electromagnetic fields stimulation on metabolic syndrome, infertility and abdominal fat-related diseases for overweight or obese patients
}

\author{
Beilin $\mathrm{G}^{1 *}$, Blanchemaison $\mathrm{P}^{2}$, Imholz $\mathrm{B}^{3}$, Abi Ghosn $\mathrm{H}^{4}, \mathrm{Al}$ Marush $\mathrm{S}^{5}$ and Haddad $\mathrm{R}^{6}$ \\ ${ }^{1}$ Aesthetic Doctor, ESAAM President, Medical Director Clinic Vendome, France \\ ${ }^{2}$ Vascular doctor, Lecturer at the Faculty of Medicine, France \\ ${ }^{3}$ Nephrologist and vascular physician, ETZ Hospital, Netherlands \\ ${ }^{4}$ Aesthetic Doctor BEYROUTH, Lebanon, Medical Trainer at Royal College, UK \\ ${ }^{5}$ Plastic surgeon, Dubai London Clinic \& Speciality Hospital, UAE \\ ${ }^{6}$ Obstetrics and Gynecology, Aesthetic \& Cosmetic Gynecology, UAE
}

\begin{abstract}
Introduction: According to various researches, abdominal obesity increases health risks.

Objective: To evaluate the reduction of waistline and the evolution of biological parameters on overweight or obese patients after 12 sessions of an electromagnetic fields medical device Class IIa.

Methods: Two studies were made: a functional study on fertility of overweight female patients performed on 38 patients candidate for an IVF (1st or 2nd attempt) with a BMI > $25 \mathrm{Kg} / \mathrm{m} 2$ for a period of 6 weeks; a scanner imaging study performed on 20 patients showing proven overweight.

Results: Following our studies after a 12 -session cure, clinical pregnancy rates after IVF in an obese infertile population of $55.55 \%$ has been observed and CT scans showed an average decrease in the visceral fat of $8,2 \%$ and to a lesser extent a decrease of subcutaneous fat of $4,1 \%$.

Conclusions: Facing an alarming increase in overweight and obesity and considering the current therapeutic gear offering various results, it seems interesting to focus on health benefits of Electromagnetic fields, also called BioEnergetic fields. The clinical studies demonstrated a positive impact on ovogenesis that might be in certain circumstances an answer to overweight-related infertility issues and a positive effect on metabolic syndrome, weight loss, visceral fat and waistline reduction of overweight or obese individuals. The clinical studies demonstrated the safety and efficiency of the devices.
\end{abstract}

\section{Introduction}

Abdominal obesity keeps increasing worldwide. According to a survey of bodyshapes conducted in the UK in 1951, a woman's average waistline was $70 \mathrm{~cm}$. A 3-D survey carried out by SizeUK in 2004 found the average woman had a waist measurement of $86 \mathrm{~cm}$.

Experts are worried that the increase in waistline and obesity will lead to an increased number of health problems. Obesity and excess weight are currently real public health problems affecting 10 to $25 \%$ of women in developed countries. As obesity and excess weight are independent factors in fertility, in particular as they affect the abdominal muscles, they must be addressed before any fertility treatment is undertaken.

The waistline is also revealing of the location of visceral fat which plays a key-role in cardio- vascular or cancer associated pathologies [1].

It is one of the major determining factors of the metabolic syndrome [2].

The basic principles for the reduction of visceral fat are: a regular physical activity, a healthier diet, a reduced consumption of salt, a decreased consumption of carbohydrates and a reduction of the waistline.
The use of BioStimology, a unique cell activation technique using alternative low frequency Electromagnetic fields to stimulate the reduction of adipose cells and to improve fertility is a genuine technique that was first developed in the 1990's [3].

\section{Material and methods}

\section{A patented technology}

The common technology, called BioStimology, is the diffusion of a low frequency alternating magnetic field oscillating from 0 to 2 Gauss. BioStimology targets smooth and striated muscle cells by activation of the calcic ATPase [4] of the sarcoplasmic reticulum (calcium pump) (Figures 1 and 2).

*Correspondence to: Ghislaine Beilin, 39 rue de Lisbonne, 75008 Paris, France, Tel. +33142940243, E-mail: gis.beilin@orange.fr

Key words: electromagnetic fields, abdominal fat, visceral fat, metabolic syndrome, obesity, fertility

Received: August 08, 2018; Accepted: August 27, 2018; Published: August 30 2018 


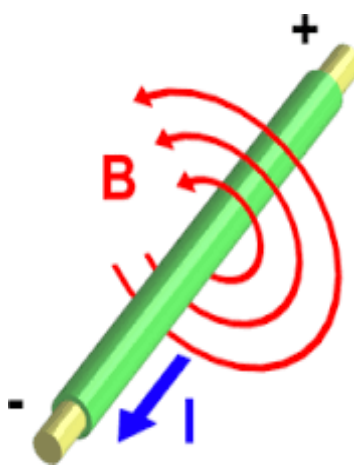

Figure 1. BioStimology

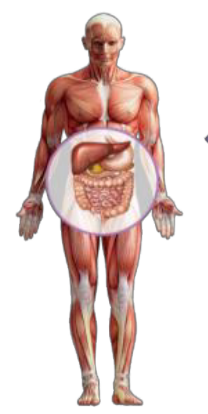

Energy expenditure by involuntary, imperceptible muscle contraction $(30 \%$ to $40 \%$ of total energy expenditure).

Elimination of visceral fat
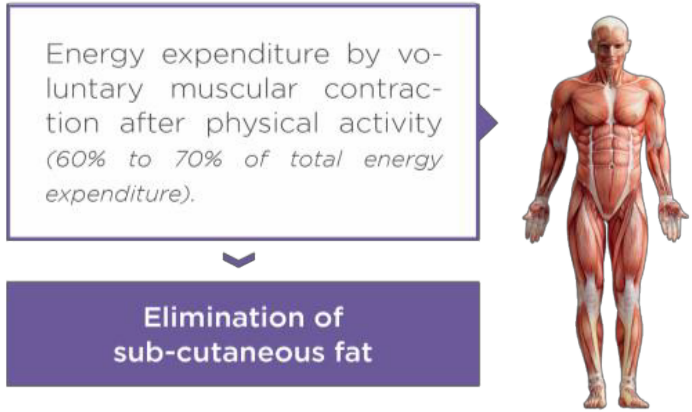

Figure 2. Muscle cell energy expenditure schema

The Electromagnetic fields induces imperceptible muscular contractions at abdominal level, similar to those resulting from sustained physical exercise, causing lipolysis of abdominal fat.

The material is presented in the form of a device (called "REDUSTIM") connected to a total body suit combining Electromagnetic fields and micropressure (pressotherapy) (Figure 3).

The biological mode of action has been decrypted in a functional analysis study performed by Doctor Philippe Benech [5] from Prediguard Laboratories Inc. intended to identify, thanks to DNA chips technology, the potential effects of 12 sessions of tested equipment, on the activity of genes expressed in the peripheral blood of 11 subjects.

As a result, at the abdominal level, the magnetic fields trigger a stimulation of insensitive muscle contraction (calcium channels) which increases the production of Lipase (HSL), thus resulting in the hydrolysis of triglycerides at the intramuscular level.

The medical indications are global management of overweight and abdominal obesity, metabolic syndrome as a factor of risk in cardiovascular diseases and strokes, infertility in obese persons, treatment of post-natal obesity, body reshaping, anti-aging prevention (Figure 4).

\section{Protocol}

In 2011, a functional study on fertility [6] (study called "Fertistim") was edited by Dr. Renato Fanchin (PMA Department - Hôpital Antoine Béclère Clamart) and performed by Dr. Vanessa Gallot and PhD René Frydman.

38 volunteers who were candidates for IVF treatment (1st or 2nd attempt) with a BMI $>25 \mathrm{Kg} / \mathrm{m}^{2}$ participated in this study and were divided into four groups according to their BMI: Overweight $(25<\mathrm{BMI}<29.9 ; \mathrm{n}=11)$, Class 1 obesity $(30<\mathrm{IMC}<34.9 ; \mathrm{n}=13)$, Class 2 obesity $(35<\mathrm{IMC}<39.9 ; \mathrm{n}=8)$ and Class 3 obesity (IMC $>40 ; n=6)$. Each patient was given 12 x 30 minute sessions of REDUSTIM $^{\oplus}$ ( 2 sessions

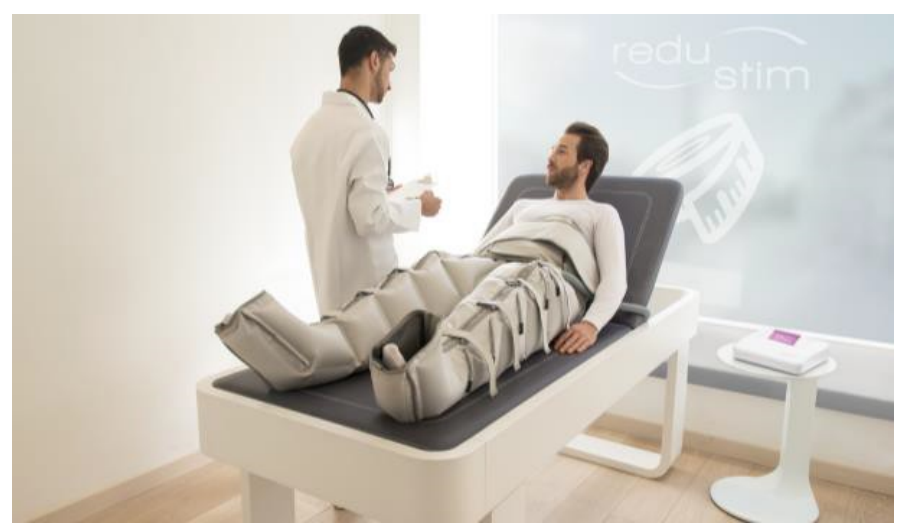

Figure 3. REDUSTIM ${ }^{\circledR}$ pressotherapy

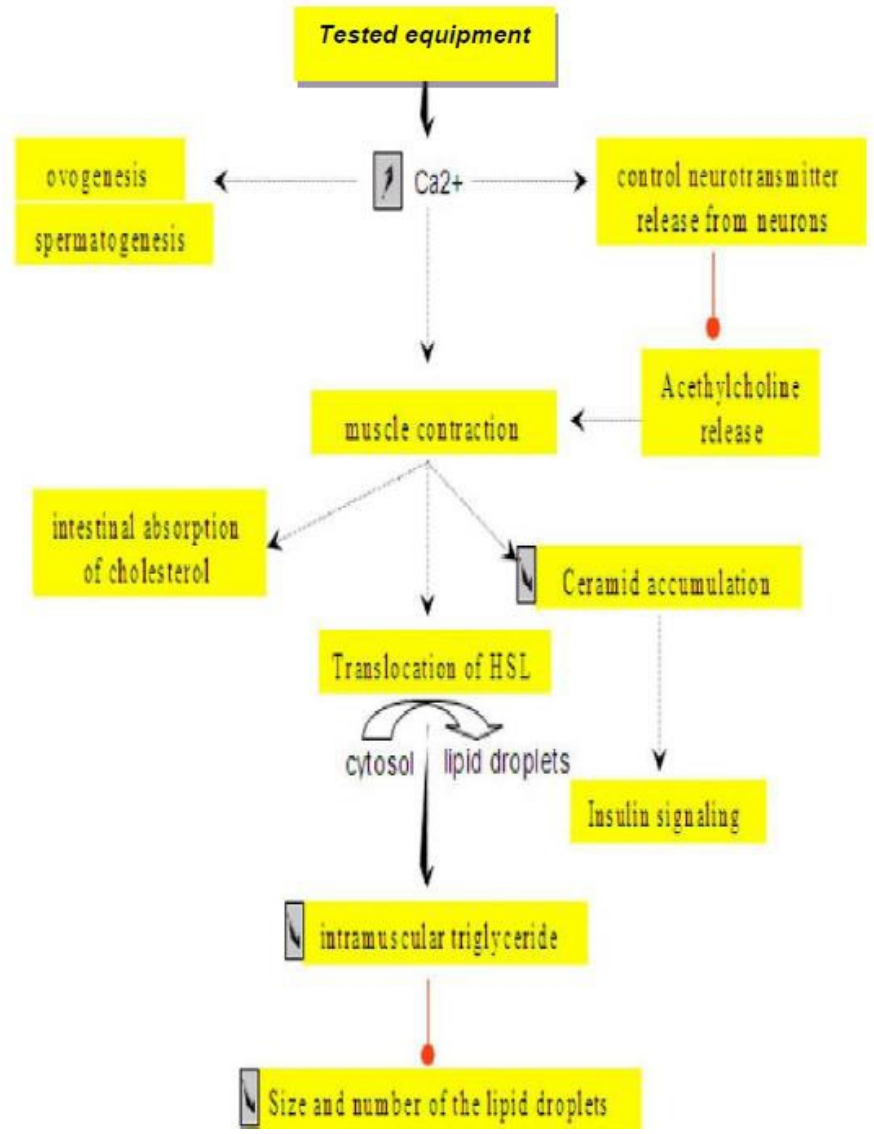

Figure 4. Biological mode of action of the electromagnetic fields technique 
per week) for 6 weeks before ovarian simulation for IVF, with no modifications to the usual clinical protocol.

In 2013, a 4-week observational scanner imaging trial [7] with REDUSTIM" SPORT (study called "Scanner"), 3 sessions a week were administered on 20 patients with an average BMI of 30.90, comparing patient quantification and comparison of the subcutaneous fat and visceral fat content before and after the study, at the level of the umbilicus, by scanner snapshot without preparation (Toshiba aquilion 16). The weight and waist circumference have also been compared before and after the treatment and a biological analysis of the ASAT and ALAT was also carried out before and after the treatment in order to review the impact of the device on the liver.

\section{Results and discussion}

\section{Mode of action}

1. One main action based on the diffusion of alternate lowfrequency electromagnetic fields in order to stimulate the fatty mass destocking. These waves present very particular properties which confers them a specific role on a lipolytic plan.

Indeed, several international studies highlighted a stimulation of membrane ATPases directly linked to calcium regulation, (usually called calcium pumps), in the presence of weak alternate magnetic fields (the low level of magnetic fields allows the implementation of the device without any risks for human body cells, or any medical operating constraints), lower than 5 Gauss (corresponding to those of the device), and this at low electrical frequencies of 40 to $60 \mathrm{~Hz}(50 \mathrm{~Hz}$ for the device).

Ca2+ ATPases are membrane enzymes located in the sarcoplasmic reticulum membrane of muscle cells where they represent $90 \%$ of membrane proteins.

Sarcoplasmic reticulum stores $\mathrm{Ca}^{2+}$ ions and the quick flow of these ions from the reticulum towards the sarcoplasm (muscle fibers cytoplasm) results in muscle contraction.

The magnetic stimulation of $\mathrm{Ca}^{2+}$ ions which results in an increased ATPases activity therefore leads to the stimulation of the lipolytic activity towards fat cells stored at the muscular level, without any consumption of additional efforts.

The action thus consists in performing part of the energy expenditure cycle, but without making demands on muscular fibers, as if a physical activity was performed, but without any muscles fibers extension.

Moreover, the excess of ATP produced by this stimulation will be eliminated by the body, in the same way as a sportsman would do it during a recovery cycle after an effort (Figure 5).

2. A secondary draining action of controlled cutaneous pressure / micropressure in order to facilitate the evacuation of fatty acids through systemic circulation and toxins elimination (Figure 6).

\section{Treatment tolerance}

Biological analysis of transaminases ASAT and ALAT were performed in the Scanner study before and after treatment to examine the impact of the technology on the liver. Transaminases ASAT and ALAT dropped by $17.4 \%$ and $18 \%$ respectively during treatment for a significance level of $\mathrm{p}<0.01$.

There is therefore no accumulation of fatty acids at the level of the liver during lipolysis, but metabolism occurs to liberate energy, carbon dioxide and water.

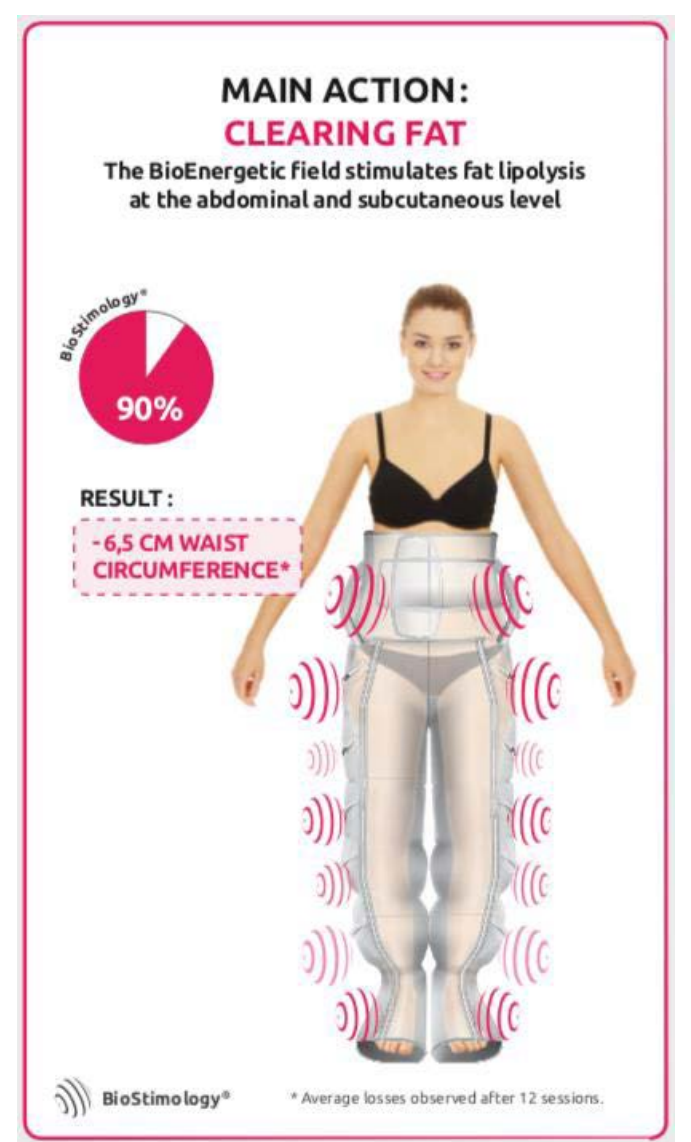

Figure 5. Schematic representation of the electromagnetic fields action

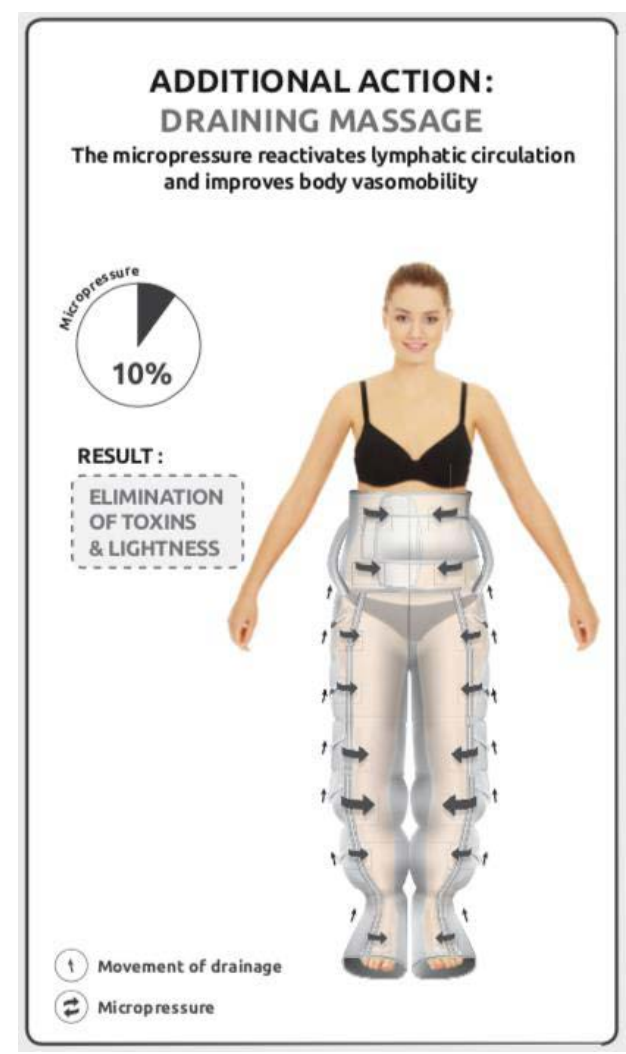

Figure 6. Schematic representation of the micropressure action 
These results show that Electromagnetic fields are particularly effective at reducing visceral fat and, to a lesser extent, subcutaneous fat.

The other blood parameters, as well as blood pressure, didn't change statistically. Furthermore, no significative side effects were noticed during this study test. In the other study on infertility, no side effects were noticed, and the satisfaction rate was good. The method has been judged non-restrictive, fast, pleasant and with no secondary effects. For these reasons, the women welcomed this alternative over all other currently existing techniques.

\section{Evolution of pregnancy}

In infertility study, an average reduction in waist size of $4.5 \mathrm{~cm}$ is observed in all the patients treated and satisfactory clinical pregnancy rates $(55.5 \%$ in total) in the four groups:

- Overweight: 4 clinical pregnancies $>36.36 \%$,

- Class 1 obesity: 6 clinical pregnancies $>46.1 \%$,

- Class 2 clinical obesity: 5 clinical pregnancies $>62.5 \%$

- Class 3 obesity: 4 clinical pregnancies $>66.7 \%$. (Figure 7).

Patient characteristics $(n=10)$ are described in Annex 1. Median age of the women studied was 32 years, $85.6 \mathrm{Kg}$ in weight and 1.61 $\mathrm{cm}$ tall. As regards the ovarian reserve, median AFC is 26 with AMH dose of $4.59 \mathrm{ng} / \mathrm{mL}$. Median BMI for the study population of women is 34 (26.7-41.7). The study population is therefore generally young with good ovarian reserve parameters and no other criteria for infertility except excess weight.

Variations in body weight and waist size during the sessions are shown in Annex 2. Median weight loss at the start of the sixth session was $1.2 \mathrm{~kg}$ then $1.6 \mathrm{~kg}$ at the end of all the sessions. The main variations in weight took place during the first 3 weeks. Changes in waist circumference followed a similar trend. Waist circumference at S6 showed a $3.0 \mathrm{~cm}$ reduction and then a $5.5 \mathrm{~cm}$ reduction at $\mathrm{S} 12$.

Annex 3 shows the results for ovarian response to stimulation and embryology. All the study parameters show improvements in ovarian response to stimulation and in the number of embryos obtained during the second IVF cycle.

Variation in weight over 6 weeks is modest $(-1.6 \mathrm{~kg})$ but it was obtained without a low-calorie diet or any particular constraints. The Fertistim study has shown the feasibility and effectiveness of the method

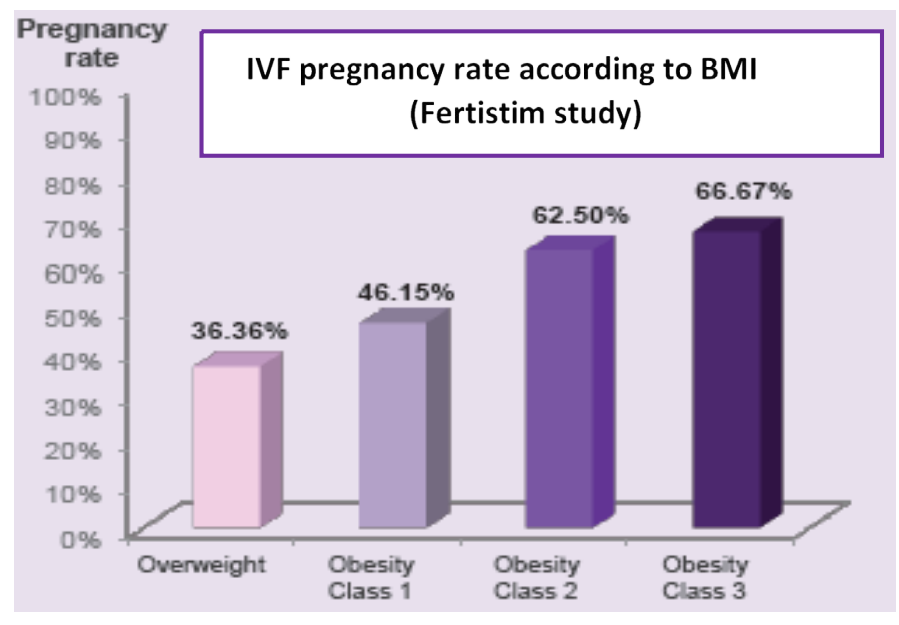

Figure 7. Evolution of pregnancy
Annex 1. Population Characteristics (Fertistim study)

\begin{tabular}{|l|l|}
\hline & Median \\
\hline Age (years) & $32(25-40)$ \\
\hline AMH $(\mathrm{ng} / \mathrm{ml})$ & $4.59(0.48-16)$ \\
\hline AFC & $26(9-60)$ \\
\hline Start weight $(\mathrm{kg})$ & $85.6(74.4-106.8)$ \\
\hline Height $(\mathrm{cm})$ & $1.61(1.53-1.71)$ \\
\hline
\end{tabular}

(Minimum and maximum values shown between brackets)

Annex 2. Variation in body weight and waist circumference (Fertistim study)

\begin{tabular}{|l|l|l|}
\hline & $\begin{array}{l}\text { Variation from } \\
\text { S0 to S6 }\end{array}$ & $\begin{array}{l}\text { Variation from } \\
\text { S0 to S12 }\end{array}$ \\
\hline Weight $(\mathrm{kg})$ & $-1.2(0-3.5)$ & $-1.6(0.5-5.0)$ \\
\hline Waist circumference & $-3.0(1.0-5.0)$ & $-5.5(0-8.0)$ \\
\hline
\end{tabular}

(Minimum and maximum values shown between brackets)

Annex 3. Ovarian response to stimulation and embryology results (Fertistim study)

\begin{tabular}{|l|l|l|l|}
\hline \multicolumn{1}{|c|}{$\mathrm{C} 1(\mathrm{n}=5)$} & $\mathrm{C} 2(\mathrm{n}=5)$ & $\mathrm{p}$ \\
\hline Number of follicles $>16 \mathrm{~mm}$ JHCG & $6.2 \pm 1.3$ & $6.6 \pm 1.8$ & $\mathrm{P}=0.423$ \\
\hline Number of ovocytes retrieved on puncture & $10.4 \pm 4.8$ & $12.4 \pm 3.4$ & $\mathrm{P}=0.273$ \\
\hline Number of embryos obtained & $4.6 \pm 2.1$ & $6.0 \pm 2.4$ & $\mathrm{P}=0.106$ \\
\hline
\end{tabular}

associating electromagnetic field and micropressure for reducing weight and waist circumference. This method is non-restrictive, fast, pleasant and has no secondary effects.

Furthermore, ovarian stimulation results and pregnancy rates after the sessions are very satisfactory. In fact, this patient population usually presents a significant failure rate in ovarian stimulation as can be seen from the patients in the first cycle, it explains why these results are very promising.

\section{Decrease of visceral fat}

In scanner study, the main goal of this study was to evaluate the ability of the treatment to achieve a decrease either in subcutaneous and/or visceral fat.

After 12 sessions this study of 20 volunteers $(12 \mathrm{~F}+8 \mathrm{M})$ with average weight at $\mathrm{S} 0$ of $95.30 \mathrm{~kg}$ and average BMI at S0 of 30.90 has shown $(\mathrm{P}<0.01)$ :

- An average reduction in weight of $5.45 \mathrm{~kg}$

- An average reduction in BMI of 6\%

- An average reduction in waistline of $7 \mathrm{~cm}$

These results confirm the findings of previous studies. However, note that previous studies used the REDUSTIM ${ }^{\circledR}$ device fitted with a micropressure system whereas in this trial the device was not equipped with such a system (other device version called "REDUSTIM" SPORT")

Hence the observed effect stems solely from diffused electromagnetic fields, not micropressure.

Concerning the main objective of this study, that is to study the impact of the device on visceral and subcutaneous fat, it seems that:

- there is a highly significant $8.2 \%$ reduction in visceral fat $(\mathrm{P}<$ $0.01)$,

- and there is a reduction in subcutaneous fat with a lower significance of $4.1 \%(\alpha=0.08)$ (Figure 8$)$. 


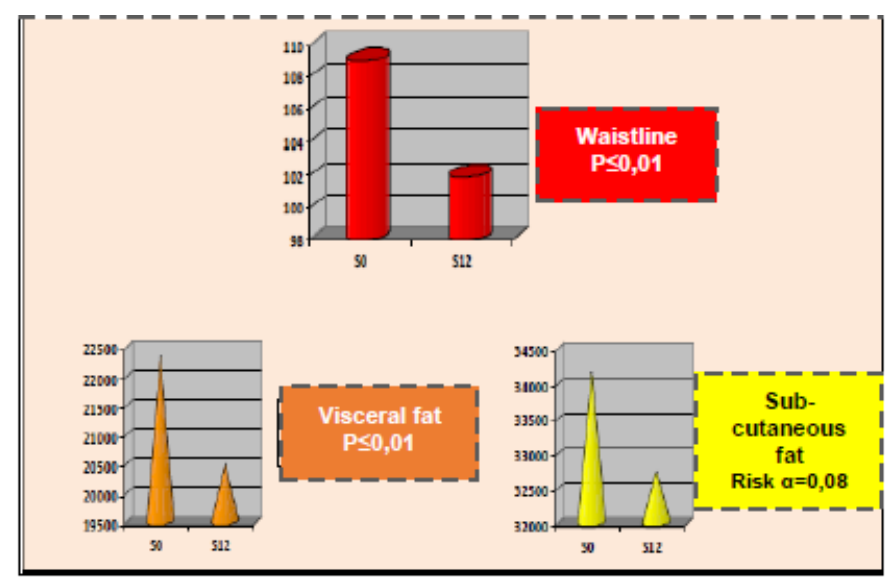

Figure 8. Comparative statistical results (Scanner study)

Transaminases ASAT and ALAT also dropped by $17.4 \%$ and $18 \%$ respectively during treatment for a significance level of $\mathrm{p}<0.01$.

There is therefore no accumulation of fatty acids at the level of the liver during lipolysis, but metabolism occurs to liberate energy, carbon dioxide and water.

These results show that the device is particularly effective at reducing visceral fat and, to a lesser extent, subcutaneous fat (Figure 9).

However, use of the device in static mode (patient lies on a treatment table and makes no physical effort) activates smooth muscles in the abdominal cavity in particular. fat.

That is why the reduction in visceral fat is twice that of subcutaneous

\section{Discussion}

After having noticed in previous study that the treatment was allowing patients to take out of the metabolic syndrome and to reduce their waistline [8], those two new studies has demonstrate other positive effects of the technology: the efficiency in the pregnancy rate on obese or overweight patients; the reduction of visceral fat for people suffering from abdominal obesity; the safety of the treatment and the good tolerance of the device.

To go even further in researching this method, and as we observe also a close relation between metabolic syndrome, abdominal fat and Diabetes Mellitus (DM), we are currently evaluating the potential extra effects of REDUSTIM ${ }^{\circledR}$ treatment besides the basic lifestyle intervention in patients with DM. A pilot study [9] (called "Diabestim") has been done by Dr. Ben Imholz in 6 patients showing on DEXA scan imaging (Annex 4) that REDUSTIM ${ }^{\circledR}$ had an effect on liver function, the overall decrease in ASAT was significant (Annex 5).

As the treatment was successful in DM patients, these results deserve to be checked on a larger cohort of subjects.

\section{Conclusion}

The results of these studies clearly demonstrated that tested equipment had a significant action on the improvement of:

1. Infertility for overweight or obese patients: increasing of pregnancy rate during IVF treatments;

2. Risk factors linked to metabolic syndrome as well as a visceral fat reduction for a positive action on lipids abnormally accumulated around internal organs or in muscles (especially on the waistline);

3. Condition of newly diagnosed patients with diabetes;

4. Healthcare prevention in a more general way including:

- Management of abdominal fat-related diseases,

- Treatment for military patient subject to abdominal obesity (a pilot study is currently in progress in the GCC zone),

- Management of overweight women looking for body reshaping: post-partum or post- menopause conditions.

The interest of BioStimology, which uses electromagnetic fields, is therefore twofold:

- On the one hand, this technology, whose mechanism of action is perfectly demonstrated, is based on muscle stimulation, without any destructive cellular process.

- On the other hand, this technology poses no risk for the skin or for the tissues since it is completely harmless and corresponds to a natural stimulation.

In an international context where regulation is more and more restrictive in order to protect users as much as possible, and where overweight and obesity represent a major economic and social challenge with a decisive impact on morbidity, it is clear that BioStimology presents the greatest future in the prevention and treatment of diseases related to excess fat.
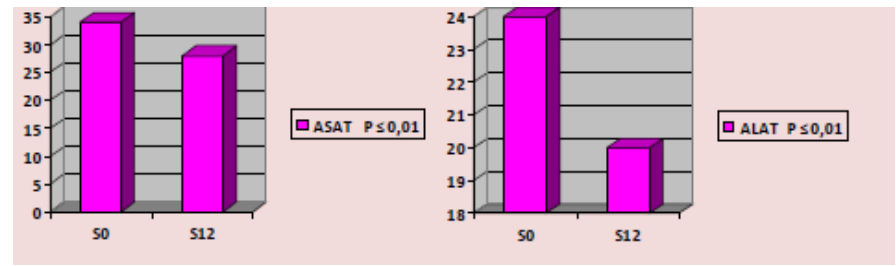

Figure 9. Transaminases results (Scanner study)

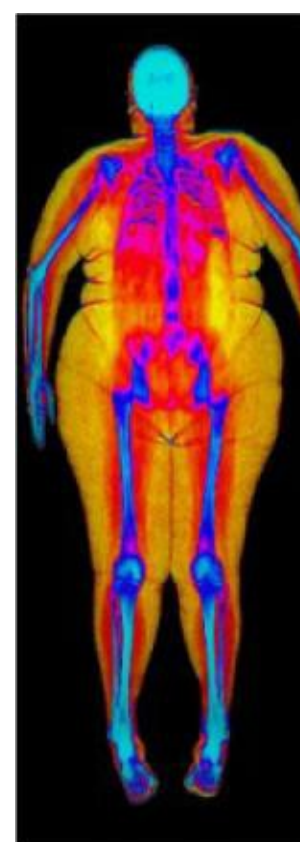

23.11.2017

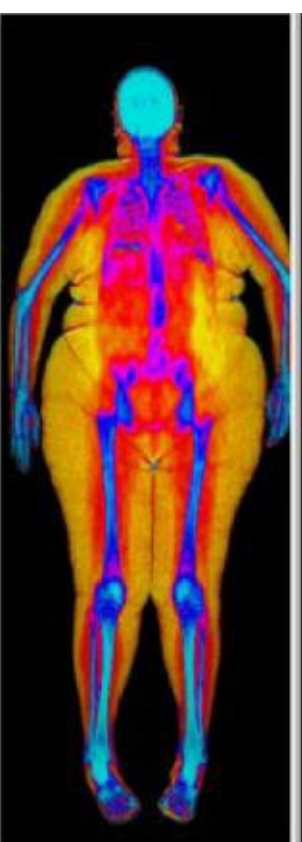

05.01.2018

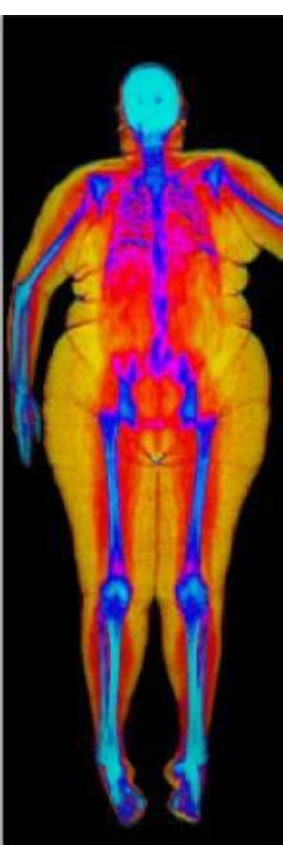

23.02 .2018
Annex 4. Example of Dexa scan imaging (Diabestim study) 


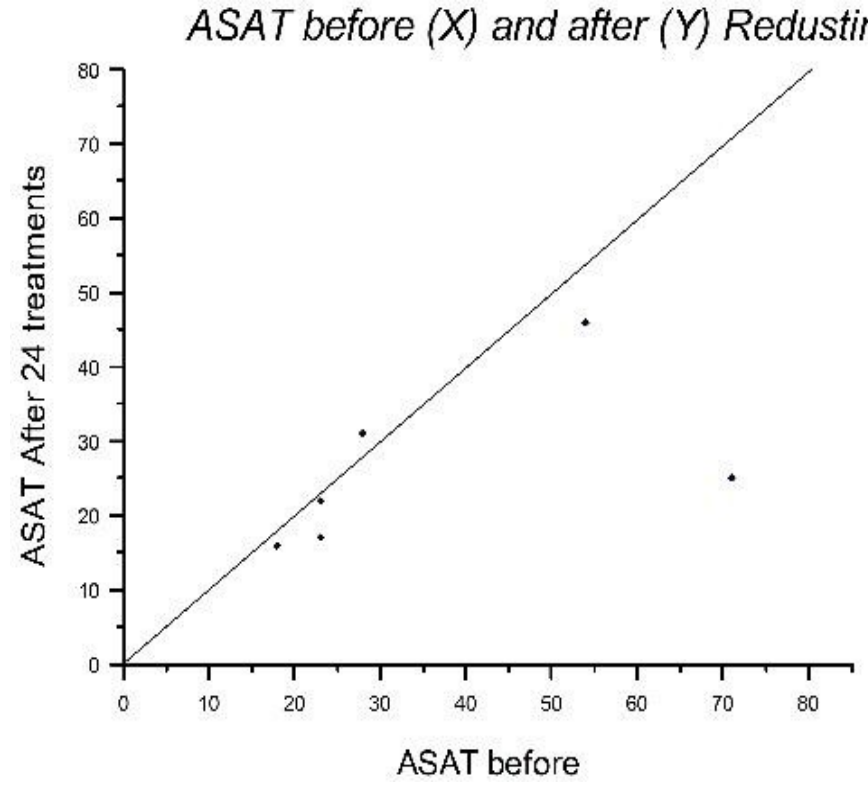

Annex 5. Changes in ASAT (Diabestim study)

\section{Acknowledgements}

This work has been done thanks to the study sponsor, Cosmosoft Laboratory, with the loan of the equipment called REDUSTIM ${ }^{\circ}$.

\section{References}

1. Rader DJ (2007) Effect of insulin resistance, dyslipidemia, and intra-abdominal adiposity on the development of cardiovascular diseases and diabetes mellitus. $\mathrm{Am} \mathrm{J}$ Med 120: S12-18. [Crossref]

2. Pischon T (2006) Body size and risk of colon and rectal cancers in the European Prospective Investigation into Cancer and Nutrition (EPIC). J Natl Cancer Inst 98: 920-931. [Crossref]

3. Comfort Harmony (1998) Development of a whole product portfolio around BioStimology technique which enabled the development of patented medical devices.

4. Blank M, L Soo, V Papstein (2005) Demonstration that a comparable magnetic field of frequency between 0 and $70 \mathrm{~Hz}$ and varying $0-2$ gauss allowed to increase the activity of the ATPases $\mathrm{Na}^{+}$and $\mathrm{K}^{+}$, (completely similar in the ATPases $\mathrm{Ca}^{++}$) from 5 to $10 \%$, that is a level of stimulation comparable to the calcic ATPases.

5. Prediguard Laboratories (2009) Functional Analysis on DNA Chips - Expression profile established to characterize the transcription effects induced in total blood cells from 11 patients treated with sessions of REDUSTIM®.

6. Frydman R, Gallot V, Fanchin R (2011) Functional study on fertility - IVF results on 38 obese and overweight patients after a reduction in waistline.

7. Nouira H, Carpentier B (2013) Scanner imaging study - Evaluation of the BioMagnetic Field utilization impact on the body fat content after 12 sessions of 30 minutes.

8. Beilin G, Benech P, Courie R, Benichoux F (2012) Electromagnetic fields applied to the reduction of abdominal obesity, PubMed ID 22171794. J Cosmet Laser Ther 14: 24-42. [Crossref]

9. Imholz B (2018) Observational pilot study - Evaluation of the potential extra effects of ReduStim treatment besides the basic lifestyle interventions in 6 patients with diabetes.

Copyright: (C2018 Beilin G. This is an open-access article distributed under the terms of the Creative Commons Attribution License, which permits unrestricted use, distribution, and reproduction in any medium, provided the original author and source are credited. 